\title{
High sensitivity optical coherence detector optimization
}

\author{
R.C. Coutinho, D.R. Selviah, H.A. French and H.D. Griffiths \\ Department of Electronic and Electrical Engineering, University College London, Torrington Place, London WC1E 7JE, United Kingdom \\ Tel.+4420 76793056, fax+4420 73889325, d.selviah@ee.ucl.ac.uk
}

\begin{abstract}
An optimal optical filtering scheme for an optical coherence detection system gives very high detection sensitivities $(-30 \mathrm{~dB})$ for a partially coherent source. Experimental results have good agreement with theoretical predictions.

(C) 2000 Optical Society of America

OCIS codes: (200.4740) Optical processing; (030.1640) Coherence; (040.1880) Detection.
\end{abstract}

\section{Introduction}

Free-space optical detection systems, such as Infrared Search and Track (IRST), measure the intensity contrast between a target and its background and apply signal processing algorithms to achieve the maximum suppression of the background and contrast enhancement. These algorithms are usually based on spatial, temporal or spatiotemporal processing [1] and, more recently, on multi-band and track-before-detect techniques [2]. In these systems, very little optical processing, if any, is employed before detection, so information such as coherence and polarization is lost in the process. In this paper we present the optimization of a technique based on the target-to-background coherence contrast. It combines pre-detector coherent optical processing with post-detector electronic signal processing using a feature extraction algorithm to give very high detection sensitivities.

The key components of this approach [3,4] are an input interference filter that creates a recognizable feature in an interferogram, an interferometer to generate the interferogram, and an algorithm to locate the feature. The method has some resemblance to Fourier Transform Spectroscopy (FTS) [5], with the advantages that the path difference scan need only be done in the neighborhood of the feature, and no Fourier transform is required. If the filter has a rectangular frequency response, then by the Fourier transform relationship between the self-coherence function (interferogram) and the power spectrum, this gives a sinc shaped envelope to the interferogram. The displacement of the first null (which occurs at the reciprocal of the filter bandwidth from zero path difference) along the path difference axis depends on the target-to-background coherence contrast.

\section{Simple Theory}

A simplified model has a target with a Gaussian spectrum and a rectangular filtered background spectrum. It can be shown that the normalized self-coherence function $\left(\Gamma_{\mathrm{N}}\right)$ of the filtered target and background radiation is given by:

$$
\Gamma_{\mathrm{N}}(\tau)=\left(\operatorname{sinc}(\pi \tau . \Delta \kappa)+P R \cdot e^{-\frac{\pi^{2}}{4 \ln 2} \delta^{2} \tau^{2}} \cdot \operatorname{erf}\left(1.176381 \frac{\Delta \kappa}{\delta}\right)\right) \cdot e^{j .2 \pi \kappa_{0} \tau}
$$

where $\tau$ is the path difference, $\Delta \kappa$ and $\delta$ are the filtered background and target optical bandwidths in wavenumbers, $\mathrm{PR}$ is the target-to-background power ratio after filtering, erf is the error function and $\kappa_{0}$ is the central wavenumber of the target and filter passbands, assumed to be coincident. The first zero occurs when $\left|\Gamma_{\mathrm{N}}(\tau)\right|=0$, which can be solved graphically. We define the differential detection responsivity to be the amount that the null is displaced when the power ratio of the target to the background is increased as given by:

$$
\frac{\partial \tau_{N}}{\partial P R} \approx \frac{\Delta \tau_{N}}{\Delta P R}=\frac{0.645 \cdot \pi}{\Delta \kappa} \cdot e^{-\frac{\pi^{2}}{4 \ln 2} \cdot(1.43)^{2}\left(\frac{\delta}{\Delta \kappa}\right)^{2}}
$$

where $\tau_{\mathrm{N}}$ is the path difference position of the null and $\partial \tau_{\mathrm{N}}$ is the amount that it moves when the power ratio is increased by $\partial \mathrm{PR}$. The instantaneous differential responsivity has been approximated the average over the range. The maximum detection responsivity for a given target bandwidth, $\delta$, is then achieved when the target-to-filter bandwidth ratio, $(\delta / \Delta \kappa)$, is equal to 0.262 . Also the peak responsivity is always found at this ratio for all target bandwidths, $\delta$, and so represents an optimization of the detection.

\section{Bandwidth Optimization Experiment}

In order to verify the theoretical prediction a set of experiments (fig 1) was carried out in the visible using red filters. The target optical source consisted of a monochromator, with a dispersion of $1.8 \mathrm{~nm} / \mathrm{mm}$, filtering the optical output of a $250 \mathrm{~W}$ white light source with $\mathrm{T}_{\text {black body }}=3200 \mathrm{~K}$. This gave a source with a variable central wavelength and bandwidth. The monochromator's entrance and exit variable slits were set to $3 \mathrm{~mm}$ to simulate a target with an 
bandwidth of $5.4 \mathrm{~nm}$. The background was simulated with a $20 \mathrm{~W}$ tungsten-halogen bulb having a similar $\mathrm{T}_{\text {black body }}=$ $3220 \mathrm{~K}$. Coupling optics collimated the light from the target and background and combined them in a cube beamsplitter. The beam diameter was limited to $10 \mathrm{~mm}$ by an iris at the detection system's input. The radiation was filtered by the input interference filter. The self coherence function was measured by a Michelson interferometer, with one of its mirrors scanned longitudinally in a $2 \mathrm{~Hz}$, triangular manner by a computer-controlled micropositioner having a resolution of $0.1 \mu \mathrm{m}$. The fringes exiting the interferometer were focused onto a large area silicon detector. The resulting electrical signal having a carrier at approximately $150 \mathrm{~Hz}$, was then amplified by $30 \mathrm{~dB}$ using an audio amplifier, and bandpass filtered between $100 \mathrm{~Hz}$ and $1 \mathrm{kHz}$. The amplified signal was sampled at $10.24 \mathrm{kHz}$ by a digitizing oscilloscope and the interferograms transferred to a computer. Different target-to-background power ratios were obtained by attenuating the radiation from the target using different power settings for the target $250 \mathrm{~W}$ light source.

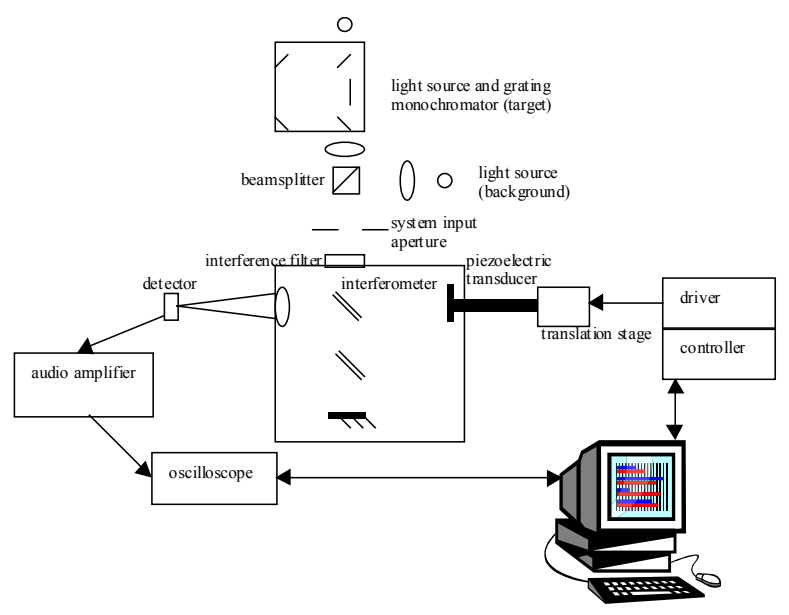

Fig. 1. Experimental arrangement

The subsequent signal processing computer algorithm removes the DC component from the data, finds the carrier frequency, and bandpass filters the interferogram using this as the central frequency. The filtered signal is phase and frequency demodulated, and the position of the feature is accurately found by detecting a step in the interferogram phase that occurs when the interferogram amplitude passes through a null $[3,4]$. Three target/filter combinations were used (Table 1). The monochromator wavelength was varied in order to give targets with central wavelengths coincident with those of the available interference filters as in the simple theory. In each set five different power ratios were tested, and for each power ratio five pairs of interferograms were recorded, each pair containing one with the target radiation blocked, and the other unblocked. The five null feature displacements were combined together to give an average and a standard deviation per point.

\begin{tabular}{ccccc}
\multicolumn{4}{c}{ Table 1. Target/filter combinations: all wavelengths and bandwidths in nm } \\
\hline Set & $\begin{array}{c}\text { Central } \\
\text { wavelength }\end{array}$ & $\begin{array}{c}\text { Target } \\
\text { bandwidth }\end{array}$ & $\begin{array}{c}\text { Filter } \\
\text { bandwidth }\end{array}$ & Ratio \\
\hline 1 & 632.6 & 5.4 & 11 & 0.491 \\
2 & 651.9 & 5.4 & 36.2 & 0.149 \\
3 & 674.8 & 5.4 & 17.8 & 0.303 \\
\hline
\end{tabular}

\section{Results and Discussion}

Figure 2 shows the three experimental points as squares and the theoretical prediction as the solid line. The experimental points follow the shape of the theoretical curve, with an optimal bandwidth ratio in the region of 0.25 0.35 . The theoretical responsivity is lower mainly because this theory used a larger range of power ratios from zero (no target present) to 1.11, where the null feature vanishes [4], whilst the experimental power ratios were in the range of 0.005 to 0.31 . The displacement versus power ratio characteristic is non-linear and so a lower responsivity would be expected for the more limited and lower range used in the experiment.

Apart from this scaling discrepancy, both curves have a peak at a similar target to background bandwidth ratio and a similar shape. One standard deviation was as much as $40 \%$ of the mean. So the experiment was repeated using a bandwidth ratio (0.303) near the peak, with the moving interferometer mirror mounted on a piezoelectric transducer which was in turn mounted on the translation stage. The mirror was scanned in a sinusoidal pattern at a frequency of $500 \mathrm{~Hz}$, with fringes at $50 \mathrm{kHz}$. This high scan rate reduced the effect of intensity fluctuations in the light sources caused by the supply and of detector $1 / \mathrm{f}$ noise but required the use of a faster silicon detector. This 
experiment gave a differential responsivity of $0.603 \mu \mathrm{m} / \mathrm{dB}$, as against 0.671 previously, but with a much-reduced standard deviation of $11.4 \%$ of the mean. Both measurements are in statistical agreement. The minimum detectable target-to-background power ratio or sensitivity, was defined as the ratio which causes an average displacement just beyond one standard deviation, was $-14.7 \mathrm{~dB}$ after filtering and $-30.3 \mathrm{~dB}$ at the input. Care must be taken in interpreting this as some noise extends beyond one standard deviation. The sensitivity also depends on the degree of coherence of the source, with lasers giving a sensitivity of $-44 \mathrm{~dB}[3,4]$. The simple theoretical model assumed that the target and filtered background spectra had no central wavelength offset. An experimental investigation was also carried out to assess the effects of a wavelength de-tuning between them. The filter in set no. 3 in table 1 was used, as it was closest to the peak responsivity in the earlier aligned wavelength experiment. Different target central wavelengths were achieved by turning the monochromator grating. The results are shown in figure 3 .

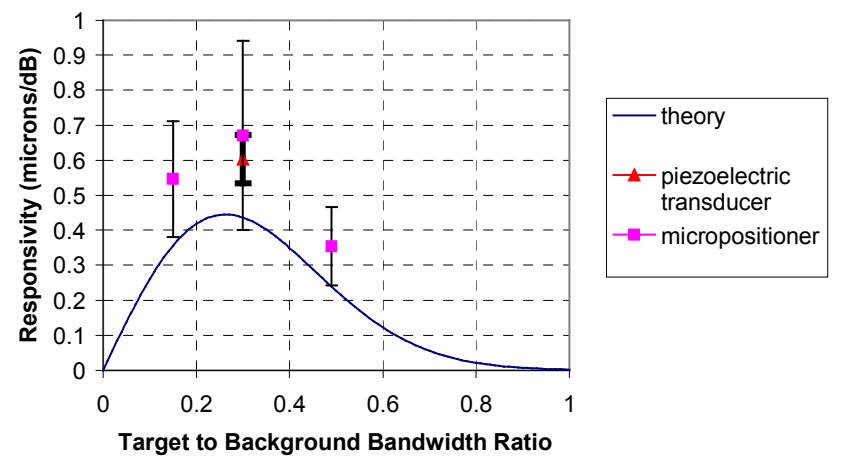

Fig. 2. Theoretical and experimental detection responsivities. The point obtained with a piezoelectric transducer and its error bars (thicker) lie within the corresponding error bars obtained with a micropositioner.

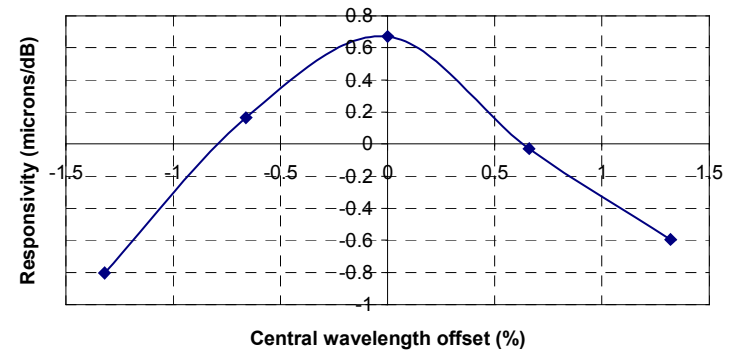

Fig. 3. Effect of target-to-filter central wavelength offset in detection responsivity

At offsets of $0.6 \%$ ( $4.5 \mathrm{~nm}$ in $674.8 \mathrm{~nm})$ the responsivity reduces to zero. Further offsets cause the displacement to become negative. This represents a reduction in coherence, as now the energy from the target is adding to the rolloff band of the filter, making the overall bandwidth larger, and the self coherence function smaller, as they are inversely proportional. The responsivity can be severely degraded if the prospective target central wavelength is not accurately centered in the optical filter spectral response, hence the penalty for achieving such high sensitivity is the need for previous knowledge of the target spectral characteristics. It remains to be established whether the optimum target to background bandwidth ratio remains optimum as the offset increases. Finally, the authors are grateful to D. Wickramasinghe and DERA Portsdown West for supplying experimental equipment and to the Brazilian Navy for extending the sabbatical leave of the first author.

\section{References}

1. S.B. Campana, ed., Passive Electro-Optical Systems, vol. 5 of The Infrared and Electro-Optical Systems Handbook (ERIM/SPIE Press, 1993), chap. 4.

2. T. Peli, P. Monsen, R. Stahl, M. Pauli and K. McCamey, "Signal processing improvements for infrared missile warning sensors", in Proceedings of the 1997 National Aerospace and Electronics Conference, vol. 2, (Institute of Electrical and Electronics Engineers, New York, 1997), pp. 1052-1064.

3. R.C. Coutinho, H.A. French, D.R. Selviah, D. Wickramasinghe, H.D. Griffiths, "Detection of coherent light in an incoherent background", in Proceedings of the IEEE Lasers and Electro-Optics Society $12^{\text {th }}$ Annual Meeting, vol. 1, (Institute of Electrical and Electronics Engineers, New York, 1999), pp. 247-248.

4. R.C. Coutinho, D.R. Selviah, H.A. French, "Detection of partially coherent optical emission sources", in Optical Pattern Recognition XI, D.P.Casasent and T. Chao, eds., Proc. SPIE 4043, 238-248 (2000).

5. J.E. Chamberlain, The Principles of Interferometric Spectroscopy (Wiley-Interscience, 1979). 